\title{
Effect of miRNA-19a on gastrointestinal motility in rats with functional dyspepsia
}

\author{
YINZHI DENG, XIANGYU ZHOU, XINGCHAO XIANG, YANGLI OU and JIANHUA HE
}

Department of Gastroenterology, The Central Hospital of Enshi Autonomous Prefecture, Enshi, Hubei 445000, P.R. China

Received December 3, 2017; Accepted February 1, 2018

DOI: $10.3892 /$ etm.2018.6009

\begin{abstract}
The effect of microRNA (miRNA)-19a on gastrointestinal motility in rats with functional dyspepsia was investigated. Fifty adult Sprague-Dawley (SD) rats were randomly divided into 5 groups, 10 rats in each group, one group as the normal group, one group as the model group, and the other three groups were divided into negative control group, miRNA-19a mimic group and miRNA-19a inhibitor group. All rats were intraperitoneally injected with miRNA-19a scramble, miRNA-19a mimic and miRNA-19a inhibitor. Except the normal group, the functional dyspepsia model rat was established by proper clipping tail stimulation. The gastric emptying rate, intestinal propulsive ratio, serum motilin and vasoactive intestinal peptide of rats in each group were measured. The level of miRNA-19a expression in each group was detected by reverse transcription-polymerase chain reaction (RT-PCR). The gastric emptying rate, intestinal propulsive ratio and serum motilin in model group were significantly lower than those in normal group, and vasoactive intestinal peptide was higher in model group than that in normal group $(\mathrm{P}<0.05)$. The expression of miRNA-19a in model group was significantly higher than that in normal group $(\mathrm{P}<0.05)$. After intraperitoneal injection of miRNA-19a mimic, the expression of miRNA-19a was increased; gastric emptying rate, intestinal propulsive ratio and serum motilin were significantly reduced in model group, and vasoactive intestinal peptide was increased $(\mathrm{P}<0.05)$. After intraperitoneal injection of miRNA-19a inhibitor, the expression of miRNA-19a was remarkably decreased; gastric emptying rate, intestinal propulsive ratio and serum motilin were further increased in model group, and vasoactive intestinal peptide was decreased $(\mathrm{P}<0.05)$. In conclusion, the expression of miRNA-19a in rats with functional dyspepsia is higher than that in normal rats,
\end{abstract}

Correspondence to: Dr Jianhua He, Department of Gastroenterology, The Central Hospital of Enshi Autonomous Prefecture, 158 Wuyang Street, Enshi, Hubei 445000, P.R. China

E-mail: fhj4km@163.com

Key words: miRNA-19a, rats, functional dyspepsia, gastrointestinal motility and the reduced miRNA-19a expression can ameliorate the gastrointestinal motility in rats with functional dyspepsia.

\section{Introduction}

Functional dyspepsia (FD) is a clinical syndrome originated from the stomach and duodenum which displays no structural abnormalities through superior gastrointestinal endoscopy (1). The prevalence of FD in Asia is 8-23\%, and it normally occurs in young people; influenced by dietary factor, social culture and psychological factors, the main clinical symptoms include postprandial fullness, epigastric pain, epigastric burning, nausea, vomiting and eructation (2). FD is a common gastrointestinal disorder that seriously affects the quality of life and socio-economic costs. The pathogenesis of FD is uncertain, which is closely related to gastrectasis hypersensitivity, impairments in the regulation of food intake, delayed gastric emptying and neuropsychological factors $(3,4)$.

MicroRNAs (miRNAs), a small non-coding RNA molecule, play a crucial role in cell proliferation, apoptosis and differentiation as a transcriptional regulator of gene expression (5). The association between miRNAs and cancer is increasingly studied; the abnormal expression of miRNAs is correlated to the occurrence and development of gastrointestinal carcinoma $(6,7)$. The analysis of miRNA chip indicates that the expression of miR-29c in gastric carcinoma tissue is obviously downregulated, and it is related to the progression of gastric cancer (8). Recent studies have shown that miRNA-510 and miRNA-29a are involved in the pathophysiology of irritable bowel syndrome (IBS) (9-11); miRNA is not only closely associated with gastrointestinal cancer, but also plays an important role in functional gastrointestinal disorders such as IBS and FD (12-14). To investigate the role of miRNA-19a in functional dyspepsia, we established models of functional dyspepsia in rats and compared the changes in miRNA-19a and studied the possible mechanisms.

\section{Materials and methods}

Animals. Sprague-Dawley (SD) rats (8-week-old male) of clean grade, weighing $205 \pm 16.5 \mathrm{~g}$, were purchased from Nanjing Junke Biological Engineering Co., Ltd., and fed in different cages. All rats were free to eat and drink in the environment of $22 \pm 1^{\circ} \mathrm{C}$ at room temperature and $65-70 \%$ in relative humidity. The study was approved by the Ethics Committee of The 
Table I. Comparison of gastrointestinal motility between model group and normal group.

\begin{tabular}{lcccc}
\hline Group & No & $\begin{array}{c}\text { Gastric emptying } \\
\text { rate }(\%)\end{array}$ & $\begin{array}{c}\text { Intestinal propulsive } \\
\text { ratio }(\%)\end{array}$ & MTL $(\mathrm{pg} / \mathrm{ml})$ \\
\hline Normal group & 10 & $77.56 \pm 4.87$ & $65.34 \pm 4.62$ & $432.4 \pm 69.2$ \\
Model group & 10 & $59.18 \pm 5.85^{\mathrm{a}}$ & $46.83 \pm 6.92^{\mathrm{a}}$ & $316.8 \pm 73.4^{\mathrm{a}}$ \\
P-value & & $<0.001$ & 0.003 & 0.015 \\
\hline
\end{tabular}

${ }^{\mathrm{a}} \mathrm{P}<0.05$.

Central Hospital of Enshi Autonomous Prefecture (Enshi, China). All authors read and approved the final manuscript.

Materials and reagents. TRIzol, reverse-transcription kit and real-time fluorescence quantitative polymerase chain reaction (qRT-PCR) kit (Promega Corp., Madison, WI, USA), miRNA-19a, U6 specific primers (Shanghai Sangon Biological Engineering Co., Ltd., Shanghai, China), miRNA-19a sequence mimics (miRNA-19a mimic) and miRNA-19a unrelated sequence mimics (scramble mimic) (Shanghai Sangon Biological Engineering Co., Ltd.), In vivo-jetPEI ${ }^{\circledR}$ (Polyplus Transfection, Illkirch, France), rat motilin (MTL), vasoactive intestinal peptide (VIP) (Wuhan Huamei Biological Engineering Co., Ltd., Wuhan, China), real-time fluorescent quantitative PCR 7500 system (Applied Biosystems, Foster City, CA, USA).

Establishment of functional dyspepsia rat model. Fifty rats were randomly divided into normal group, model group, miRNA-19a mimic group, miRNA-19a inhibitor group, miRNA-19a scramble (negative control) group using a random number table, 10 rats in each group. Rat models of functional dyspepsia in the other groups were established by proper clipping tail stimulation except the normal group (12). During the modeling period, the feeding and drinking of the experimental animals were not restricted, and the activity, weight and food intake of the rats were observed.

$q R T-P C R$. The total RNA of tissue was extracted by TRIzol and used as a template to be reverse transcribed into cDNA. Subsequently, PCR amplification was conducted in accordance with the steps in the qRT-PCR kit, with U6 as internal reference, for 40 cycles. The relative expression level of miRNA was expressed by $2^{-\Delta \Delta \mathrm{Cq}}$. The primers of miRNA-19a were as follows: forward, 5'-CCGGTGTGCAAATCTATGCAA-3' and reverse, 5'-CAGTGCAGGGTCCGAGGTAT-3'.

Gastric emptying rate and intestinal propulsive ratio. After rats were treated by fasting, not fluid deprivation, for $24 \mathrm{~h}$, intragastric administration with semisolid paste was given. After $30 \mathrm{~min}$, rats were sacrificed, followed by anatomy and measurement of total length of small intestine (pylorus - ileocecus). The distance from the pylorus to the leading edge of the ink served as the propelling distance of the ink in the intestine. Intestinal propulsive ratio = propelling length of the ink/total length of small intestine x $100 \%$. Total gastric mass was weighed, and gastric body was cut open along with greater curvature, followed by washing away gastric content. The net gastric mass was weighed after drying. Gastric emptying rate $=[1$ - (total gastric mass - net gastric mass)/mass of intragastric administration) x100\% (13).

Serum MTL and VIP. The abdominal aortic blood was extracted and naturally coagulated, followed by centrifugation at $2,450 \mathrm{x}$ g for $15 \mathrm{~min}$ at $4^{\circ} \mathrm{C}$ and collection of serum. The serum MTL and VIP were determined according to the instructions in the enzyme-linked immunosorbent assay (ELISA) kit.

Intraperitoneal injection with miRNA-19a mimic and miRNA-19a inhibitor. According to the instructions, In vivo-jetPEI and miRNA-19a mimic, miRNA-19a inhibitor or miRNA-19a scramble were mixed at a certain proportion and injected into the abdominal cavity of the rats, once every four days. The aseptic operation needs special attention, and other operations were the same as those in the model group. The functional dyspepsia rat model was established by clipping tail stimulation.

Statistical analysis. SPSS 17.0 software (SPSS, Inc., Chicago, IL, USA) was used for statistical analysis. Measurement data are expressed as mean \pm SD. Student's t-test was adopted for independent samples. The analysis of variance was utilized for multivariate data. $\mathrm{P}<0.05$ was considered to indicate a statistically significant difference.

\section{Results}

Comparisons of gastric emptying rate, intestinal propulsive ratio, MTL and VIP between model and normal group. The gastric emptying rate and intestinal propulsive ratio between normal and model group were calculated, further suggesting that gastric emptying rate and intestinal propulsive ratio in model group were significantly lower than those in normal group $(\mathrm{P}<0.05)$. MTL and VIP in rats were calculated by ELISA, which displayed that MTL in model group was remarkably decreased, and VIP was increased $(\mathrm{P}<0.05)$, indicating that the rat model of functional dyspepsia was successfully established (Table I).

Comparison of difference of miRNA-19a expression between model and normal group. The contents of miRNA-19a in rat gastric tissue of model and normal group were detected by qRT-PCR, which displayed that the relative content of 
Table II. Effect of intraperitoneal injection with miRNA-19a mimic and miRNA-19a inhibitor on gastric motility.

\begin{tabular}{|c|c|c|c|c|c|}
\hline Group & No. & $\begin{array}{c}\text { Gastric emptying } \\
\text { rate }(\%)\end{array}$ & $\begin{array}{l}\text { Intestinal propulsive } \\
\text { ratio }(\%)\end{array}$ & MTL (pg/ml) & $\mathrm{VIP}(\mathrm{pg} / \mathrm{ml})$ \\
\hline miR-19a scramble & 10 & $55.25 \pm 5.28$ & $45.88 \pm 7.69$ & $308.45 \pm 50.34$ & $420.45 \pm 59.34$ \\
\hline miR-19a mimic & 10 & $41.98 \pm 7.24^{\mathrm{a}}$ & $32.78 \pm 8.24^{\mathrm{a}}$ & $214.34 \pm 69.23^{\mathrm{a}}$ & $500.23 \pm 68.38^{\mathrm{a}}$ \\
\hline miR-19a inhibitor & 10 & $78.62 \pm 6.35^{\mathrm{a}}$ & $68.45 \pm 7.93^{\mathrm{a}}$ & $452.63 \pm 72.24^{\mathrm{a}}$ & $306.27 \pm 72.93^{\mathrm{a}}$ \\
\hline P-value & & $<0.001$ & 0.009 & 0.002 & 0.008 \\
\hline
\end{tabular}

${ }^{\mathrm{a}} \mathrm{P}<0.05$.

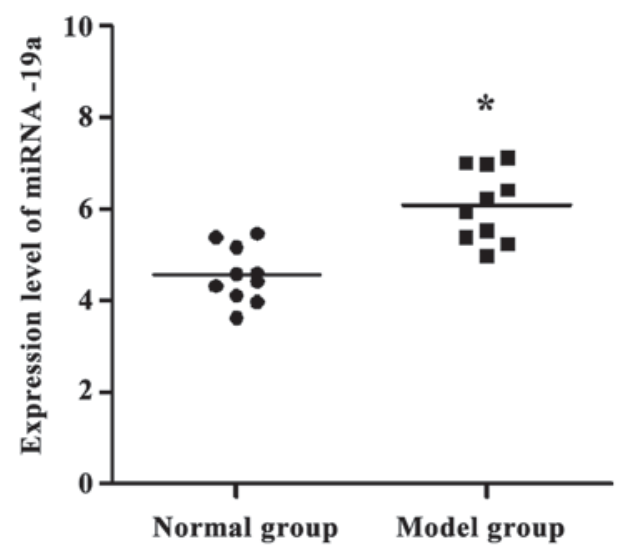

Figure 1. Comparison of difference of miRNA-19a expression between model and normal group. The contents of miRNA-19a in rat gastric tissue of model and normal group are detected by qRT-PCR, which displayed that the relative content of miRNA-19a in gastric tissue of model group is $6.13 \pm 0.89$, which is $4.56 \pm 0.65$ in normal tissue, suggesting that miRNA-19a is highly expressed in rats with functional dyspepsia, ${ }^{*} \mathrm{P}<0.05$.

miRNA-19a in gastric tissue of model group was $6.13 \pm 0.89$, which was $4.56 \pm 0.65$ in normal tissue, suggesting that miRNA-19a was highly expressed in rats with functional dyspepsia (Fig. 1).

Changes of miRNA-19a after intraperitoneal injection with miRNA-19a mimic, miRNA-19a inhibitor. During the establishment of the rat model of functional dyspepsia, the expression of miRNA-19a was $8.52 \pm 1.04$ in rats intraperitoneally injected with miRNA-19a mimic, $3.02 \pm 0.99$ in those injected with miRNA-19a inhibitor, 4.62 \pm 0.76 in miRNA-19a scramble group, indicating that the expression of miRNA-19a in rats intraperitoneally injected with miRNA-19a mimic was obviously higher than that in the negative control group, and miRNA-19a inhibitor could remarkably decrease it in the gastric tissue $(\mathrm{P}<0.05)$ (Fig. 2).

Effect of intraperitoneal injection with miRNA-19a mimic and miRNA-19a inhibitor on gastric motility. During the establishment the rat model of functional dyspepsia, the changes of gastric emptying rate, intestinal propulsive ratio, MTL and VIP in serum of rats intraperitoneally injected with miRNA-19a mimic and miRNA-19a inhibitor were further studied, indicating that gastric emptying rate, intestinal propulsive ratio and MTL in serum after injection with

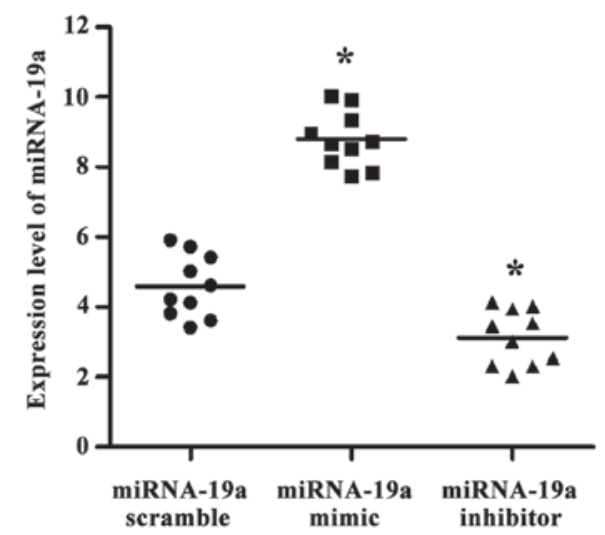

Figure 2. Changes of miRNA-19a after intraperitoneal injection with miRNA-19a mimic, miRNA-19a inhibitor. After rats are intraperitoneally injected with miRNA-19a mimic, miRNA-19a inhibitor and negative control miRNA-19a scramble in rat model of functional dyspepsia, the expression of miRNA-19a was detected, indicating that the expression of miRNA-19a in rats of miRNA-19a mimic group is obviously increased, which significantly decreased in miRNA-19a inhibitor group, ${ }^{*} \mathrm{P}<0.05$.

miRNA-19a mimic were increased $(\mathrm{P}<0.05)$. After injection with miRNA-19a inhibitor, it was found that gastric emptying rate, intestinal propulsive ratio and MTL in serum were significantly increased in model group, and VIP was decreased $(\mathrm{P}<0.05)$ (Table II).

\section{Discussion}

miRNA-19a is an important member of the miRNA-17-92 cluster. miR-17-92 gene is an oncogene, expressed in a variety of tumors, but its mechanism is unclear. The low miRNA-19a expression in serum can be used as a new indicator of poor prognosis in multiple myeloma (MM), but it is more sensitive to high expression of miRNA-19a relative to bortezomib that can reverse the prognosis of MM patients with low expression of miRNA-19a, thus improving survival time (14). miRNA-19a is highly expressed in bladder cancer (15), liver cancer (16), lung cancer (17), colon cancer (18) and other multiple tumors, which acts as an oncogene, and it is closely related to tumor invasion, metastasis, invasion and drug resistance. A study shows that miRNA-19a is highly expressed in the tissue of gastric cancer, and the high expression of miRNA-19a can induce the epithelial mesenchymal transition through phosphatidylinositol-3-kinase (PI3K)/serine/threonine kinase (Akt) pathway, promoting cell proliferation, migration and 
invasion, thus playing a role in carcinogenesis; the prognosis of patients with high miRNA-19a expression is poor (19). In gastric cancer cells, high expression levels of miRNA-19a can lead to the enhancement of cell proliferation and migration via activating nuclear factor $\kappa-\mathrm{B}(\mathrm{NF}-\kappa \mathrm{B})(20)$. The abnormal expression of miRNA is closely related with the occurrence and development of tumors; moreover, the dysregulation of miRNA expression can lead to a variety of diseases. miRNA is not only closely associated with gastrointestinal cancer, but also plays an important role in IBS, FD and other functional gastrointestinal diseases. This study aimed to investigate the effect of miRNA-19a on functional dyspepsia in rats.

The rat functional dyspepsia model was established by clipping tail stimulation, and the detection revealed that gastric emptying rate and intestinal propulsive ratio in rats of model group were significantly lower than those in normal group. MTL, as a gastrointestinal hormone, promotes gastric emptying and stimulates gastrointestinal motility through inducing the gastric three-phase of the migrating complex. In the digestive system, MTL can increase appetite and inhibit nausea and plays different roles in a variety of digestive disorders. In patients with functional dyspepsia, gastric emptying function is reduced, and gastric electrical rhythm is disordered, which are related to the decrease of plasma MTL content; gastroparesis or slow gastric emptying can be treated by MTL receptor agonist (21). VIP is not only a gastrointestinal hormone but also a neuropeptide. It is widely distributed in various body systems and closely related to and widely applied in the study of gastrointestinal diseases. The comparison of detection of MTL and VIP between model and normal group displayed that MTL was significantly lower in model group than that in normal group, and VIP was higher in model group than that in normal group $(\mathrm{P}<0.05)$. Thus, the model was established successfully.

miRNA-19a is closely related to functional dyspepsia and involved in the regulation of gastric motility in rats with functional dyspepsia. The contents of miRNA-19a in model and normal group were detected by qRT-PCR, which showed that miRNA-19a expression in model group was higher than that in normal group $(\mathrm{P}<0.05)$, indicating that miRNA-19a participates in the regulation of rat functional dyspepsia. To further determine the role of miRNA-19a, the intraperitoneal injection with miRNA-19a mimic was adopted, suggesting that gastric emptying rate, intestinal propulsive ratio and serum MTL in model group were significantly decreased, and VIP was increased $(\mathrm{P}<0.05)$; the high expression of miRNA-19a could decrease gastric motility in rats, resulting in elevating the harm of the disease. In contrast, after intraperitoneal injection with miRNA-19a inhibitor, gastric emptying rate, intestinal propulsive ratio and serum MTL were further increased in model group, and VIP was decreased $(\mathrm{P}<0.05)$. The gastric function can be ameliorated through reducing the expression of miRNA-19a.

This study indicated that miRNA-19a is highly expressed in the functional dyspepsia and can inhibit gastrointestinal motility. The gastrointestinal motility can be improved by reducing the expression of miRNA-19a.

\section{Acknowledgements}

Not applicable.

\section{Funding}

No funding was received.

\section{Availability of data and materials}

The datasets used and/or analyzed during the current study are available from the corresponding author on reasonable request.

\section{Authors' contributions}

YD wrote the manuscript. YD and XZ established functional dyspepsia rat . YD and XX conducted PCR. YO and $\mathrm{JH}$ were responsible for gastric emptying rate and intestinal propulsive ratio. All authors read and approved the final manuscript.

\section{Ethics approval and consent to participate}

The study was approved by the Ethics Committee of The Central Hospital of Enshi Autonomous Prefecture (Enshi, China).

\section{Consent for publication}

Not applicable.

\section{Competing interests}

The authors declare that they have no competing interests.

\section{References}

1. Imthon AK, Moeller ME, Drewes AM, Drewes AM, Juel J and Aziz Q: Functional gastroduodenal disorders. Hamdan Med J 8: $253-263,2015$

2. Ghoshal UC, Singh R, Chang FY, Hou X, Wong BC and Kachintorn U; Functional Dyspepsia Consensus Team of the Asian Neurogastroenterology and Motility Association and the Asian Pacific Association of Gastroenterology: Epidemiology of uninvestigated and functional dyspepsia in Asia: Facts and fiction. J Neurogastroenterol Motil 17: 235-244, 2011.

3. Zhang GS, Xie S, Hu W, Liu RE, Liu ML, Liu M and Chang XY: Effects of electroacupuncture on interstitial cells of Cajal (ICC) ultrastructure and connexin 43 protein expression in the gastrointestinal tract of functional dyspepsia (FD) rats. Med Sci Monit 22: 2021-2027, 2016

4. El-Serag HB and Talley NJ: Systemic review: The prevalence and clinical course of functional dyspepsia. Aliment Pharmacol Ther 19: 643-654, 2004

5. He L and Hannon GJ: MicroRNAs: Small RNAs with a big role in gene regulation. Nat Rev Genet 5: 522-531, 2004.

6. Wang D, Fan Z, Liu F and Zuo J: Hsa-miR-21 and Hsa-miR-29 in tissue as potential diagnostic and prognostic biomarkers for gastric cancer. Cell Physiol Biochem 37: 1454-1462, 2015.

7. Ueda T, Volinia S, Okumura H, Shimizu M, Taccioli C, Rossi S, Alder H, Liu CG, Oue N, Yasui W, et al: Relation between microRNA expression and progression and prognosis of gastric cancer: A microRNA expression analysis. Lancet Oncol 11: 136-146, 2010.

8. Saito Y, Suzuki H, Imaeda H, Matsuzaki J, Hirata K, Tsugawa H, Hibino S, Kanai Y, Saito H and Hibi T: The tumor suppressor microRNA-29c is downregulated and restored by celecoxib in human gastric cancer cells. Int J Cancer 132: 1751-1760, 2013.

9. Kapeller J, Houghton LA, Mönnikes H, Walstab J, Möller D, Bönisch H, Burwinkel B, Autschbach F, Funke B, Lasitschka F, et al: First evidence for an association of a functional variant in the microRNA-510 target site of the serotonin receptor-type 3E gene with diarrhea predominant irritable bowel syndrome. Hum Mol Genet 17: 2967-2977, 2008. 
10. Zhou Q, Souba WW, Croce CM and Verne GN: MicroRNA-29a regulates intestinal membrane permeability in patients with irritable bowel syndrome. Gut 59: 775-784, 2010.

11. Saito Y, Suzuki H, Tsugawa H, Suzuki S, Matsuzaki J, Hirata K and Hibi T: Dysfunctional gastric emptying with down-regulation of muscle-specific microRNAs in Helicobacter pylori-infected mice. Gastroenterology 140: 189-198, 2011.

12. Abdel-Aziz H, Wadie W, Zaki HF, Müller J, Kelber O, Efferth T and Khayyal MT: Novel sequential stress model for functional dyspepsia: Efficacy of the herbal preparation STW5. Phytomedicine 22: 588-595, 2015.

13. Talley NJ: Functional dyspepsia: New insights into pathogenesis and therapy. Korean J Intern Med 31: 444-456, 2016.

14. Hao M, Zang M, Wendlandt E, Xu Y, An G, Gong D, Li F, Qi F Zhang Y, Yang Y, et al: Low serum miR-19a expression as a novel poor prognostic indicator in multiple myeloma. Int J Cancer 136: $1835-1844,2015$.

15. Feng Y, Liu J, Kang Y, He Y, Liang B, Yang P and Yu Z: miR-19a acts as an oncogenic microRNA and is up-regulated in bladder cancer. J Exp Clin Cancer Res 33: 67, 2014.

16. Baik SH, Lee J, Lee YS, Jang JY and Kim CW: ANT2 shRNA downregulates miR-19a and miR-96 through the PI3K/Akt pathway and suppresses tumor growth in hepatocellular carcinoma cells. Exp Mol Med 48: e222, 2016.
17. Yamamoto K, Ito S, Hanafusa H, Shimizu K and Ouchida M: Uncovering direct targets of miR-19a involved in lung cancer progression. PLoS One 10: e0137887, 2015.

18. Huang L, Wang X, Wen C, Yang X, Song M, Chen J, Wang C, Zhang B, Wang L, Iwamoto A, et al: Hsa-miR-19a is associated with lymph metastasis and mediates the TNF- $\alpha$ induced epithelial-to-mesenchymal transition in colorectal cancer. Sci Rep 5: 13350, 2015.

19. Lu W, Xu Z, Zhang M and Zuo Y: MiR-19a promotes epithelialmesenchymal transition through PI3K/AKT pathway in gastric cancer. Int J Clin Exp Pathol 7: 7286-7296, 2014.

20. Yang F, Wang H, Jiang Z, Hu A, Chu L, Sun Y and Han J: MicroRNA-19a mediates gastric carcinoma cell proliferation through the activation of nuclear factor- $\kappa \mathrm{B}$. Mol Med Rep 12: 5780-5786, 2015.

21. Sanger GJ and Furness JB: Ghrelin and motilin receptors as drug targets for gastrointestinal disorders. Nat Rev Gastroenterol Hepatol 13: 38-48, 2016.

(i) $\ominus$ This work is licensed under a Creative Commons Attribution-NonCommercial-NoDerivatives 4.0 International (CC BY-NC-ND 4.0) License. 